msh-mss Mathématiques et sciences humaines

196 | hiver 2011

Varia

\title{
Un mesurage pour les nombres recueillis $[0,1]$ avec un curseur, au cours de présentations par couple de stimuli
}

A measurement for numbers recorded on $[0,1]$ with a cursor during the presentation of pairs of stimuli

Michel Maurin

(2) OpenEdition

Journals

Édition électronique

URL : http://journals.openedition.org/msh/12132

DOI : $10.4000 /$ msh. 12132

ISSN : $1950-6821$

Éditeur

Centre d'analyse et de mathématique sociales de l'EHESS

Édition imprimée

Date de publication : 30 décembre 2011

Pagination : 41-61

ISSN : 0987-6936

Référence électronique

Michel Maurin, « Un mesurage pour les nombres recueillis $[0,1]$ avec un curseur, au cours de

présentations par couple de stimuli », Mathématiques et sciences humaines [En ligne], 196 | hiver 2011, document 1444, mis en ligne le 15 avril 2012, consulté le 23 juillet 2020. URL : http://

journals.openedition.org/msh/12132 ; DOI : https://doi.org/10.4000/msh.12132 


\title{
UN MESURAGE POUR LES NOMBRES RECUEILLIS SUR [0,1] AVEC UN CURSEUR, AU COURS DE PRÉSENTATIONS PAR COUPLE DE STIMULI
}

\author{
Michel MAURIN ${ }^{1}$
}

\begin{abstract}
RÉSUMÉ - On dispose de nombreux procédés pour recueillir des réponses de sujets au cours des enquêtes. L'un d'entre eux consiste à positionner la réponse avec un signe sur un trait horizontal dessiné sur le questionnaire, comme une cotation graphique. Cela revient à utiliser un curseur sur le segment [0,1]. De cette manière la réponse est directement un nombre, mais un nombre qui est soumis aux effets de bord des deux bornes du continuum de ce procédé de recueil. De ce fait les opérations numériques habituelles ne peuvent convenir sur de tels nombres. L'article consiste à proposer une échelle de mesure dans le cadre de la théorie du mesurage, avec théorèmes de représentation et de caractérisation (uniqueness), et pertinence (meaningfulness) des énoncés numériques et statistiques. Les stimuli sont présentés par couple et les outils techniques utilisés sont ici le birapport et un groupe de fonctions homographiques. En dernier on observe que des transformées simples de la réponse sur [0,1] sont mesurées sur des échelles de mesure plus habituelles (échelles de rapport et de différence), et cela offre la possibilité de nombreux traitements statistiques pertinents sur ces transformées, avec les statistiques ordinaires.
\end{abstract}

MOTS-CLÉS - Birapport, Continuum borné, Cote, Couple de stimuli, Fonction homographique, Plan d'expérience, Théorie du mesurage

SUMMARY - A measurement for numbers recorded on [0,1] with a cursor during the presentation of pairs of stimuli

Many methods for recording subject's responses in surveys are available. One of them is to mark a sign on a horizontal segment of a straight line drawn in the questionnaire, as a graphical valuation. It is like the use of an individual cursor on [0,1]. In this way, the response is a number, but a number subject to important boundary effects close to the two endpoints of the continuum of possible responses, and indeed the usual and common numerical operations on numbers are not valid here. The object of this paper is to propose a measurement scale for these numbers included in the general framework of measurement theory, with a representation theorem, a uniqueness theorem, and meaningfulness of statistical statements. The stimuli are presented in pairs (two by two), and the technical tools used here are the cross-ratio and a group of homographic functions. Finally one observes that simple transforms of responses on [0,1] are measured on more usual and familiar scales of measurement (ratio and difference scales). This leads to a number of possible, common and meaningful statistical processings that can be applied to these transformed cursor-[0,1]'s responses.

KEYWORDS - Bounded continuum, Cross-ratio, Design of experiment, Homographic function, Measurement theory, Odd, Stimuli's couple

1 Institut National de Recherche sur les Transports et leur Sécurité (INRETS-LTE), Case 24, 69675 Bron cedex, michel.maurin@ifsttar.fr [Remarque : l'INRETS est devenu l'Institut Français des Sciences et Technologies des Transports de l'Aménagement et des Réseaux (IFSTTAR) en 2011]. 


\section{INTRODUCTION}

Le recueil des données dans les enquêtes et les sciences humaines revêt plusieurs formes ou modalités. Sous la pression du quantitative imperative [Michell, 1990], de la quantophrénie de Sorokin [Parain-Vial, 1981] ou autre number appeal [Maurin, 2009], on rencontre divers procédés qui ont pour effet de produire des recueils numériques de manière directe ou des recueils numérisés après transformation. On reconnaît là bien des procédés de la psychométrie de Galton et de la psychophysique de Fechner.

Le procédé examiné ci-après est le recueil de la réponse au moyen d'une marque dessinée sur un support présenté au sujet quand on lui demande de positionner sa réponse par un signe entre les deux extrémités du trait, comme une « échelle de cotation graphique » sur un continuum de réponse [Bonnet, 1986], à l'image d'un curseur sur le trait. On peut le ramener aussitôt à un nombre de l'intervalle $[0,1]$ et être ensuite tenté de travailler avec ces nombres comme on le fait de manière ordinaire en les dotant de toutes les propriétés des nombres, mais avec une certaine candeur et en ayant peut-être un peu perdu de vue leur provenance et leur mode d'acquisition.

L'usage des nombres cependant nécessite des précautions et il revient à la Théorie du Mesurage, measurement theory, d'avoir précisé et posé les règles de l'emploi des nombres dans les disciplines qui ne sont pas directement exprimées à l'aide du formalisme mathématique [Suppes, Zinnes, 1963; Roberts, 1979]. Cette théorie est véritablement apparue au cours de la seconde moitié du XXe siècle et elle s'efforce d'attribuer ou d'assigner des nombres, numerical assignment, par l'intermédiaire de l'existence d'une représentation numérique, representation, puis de caractériser l'échelle de mesurage qui en résulte, uniqueness, avant de statuer sur la pertinence des énoncés numériques qui font état de ces nombres, meaningfulness.

Cet article traite du recueil des réponses sur un continuum borné. La Section 2 fait le point sur les questions qui sont soulevées a priori par cette pratique, la Section 3 est l'étude préliminaire d'un groupe de transformations numériques qui laissent le segment $[0,1]$ invariant, la Section 4 est le cœur d'une méthode de mesurage que l'on peut appliquer à ce type de recueil (avec représentation et caractérisation), et la Section 5 aborde quelques questions liées à la pertinence. Le résultat conduit à une échelle de mesure orthodoxe comme la théorie les présente, mais avec une originalité qui peut dérouter au premier abord. Quoi qu'il en soit la Section 6 introduit de nouveaux mesurages associés qui sont sur des échelles plus abordables sur le plan pratique, et la Section 7 présente différentes possibilités vers des traitements statistiques pertinents et classiques en aval. La Section 8 et les Annexes sont l'occasion de prendre un peu de recul sur cette application particulière du mesurage et sur les outils qu'elle utilise.

Par ailleurs ces réflexions proviennent d'une question soulevée à l'occasion de discussions, elles demeurent ci-après au niveau du développement méthodologique et elles ne s'appliquent pas (encore) à des jeux de données recueillies ou récupérées à cet effet.

\section{UN PREMIER ROUND D'OBSERVATION DE LA QUESTION}

Le recueil d'une réponse sur un continuum borné par deux points possède des aspects spécifiques et, afin de les souligner, d'autres procédés de recueil sont présentés pour commencer. 


\subsection{QUELQUES RAPPELS SUR D'AUTRES PROCÉDÉS}

- Les comparaisons par paire interviennent à de nombreuses reprises. En substance on présente deux stimuli $s_{a}$ et $s_{b}$ à un sujet, et celui-ci exprime la préférence qu'il éprouve pour l'un par rapport à l'autre moyennant une probabilité ou une fréquence $p_{a b}$. La suite consiste à en déduire des valeurs numériques $x_{a}$ et $x_{b}$ sur $\mathrm{R}$, c'est notamment le cas de la loi des jugements comparatifs de Thurstone [Torgerson, 1958 ; Bonnet, 1986 ; Martin, 1997], puis de diverses mises en œuvre du mesurage dont le modèle de Bradley, Terry et Luce (BTL), [Suppes, Zinnes, 1963].

- On a le cas des réponses sur des échelles catégorielles ordonnées $C_{j}, j=1 \ldots J$, lorsque le nombre de stimuli $s_{a}$ devient important; dans ce cadre le sujet doit placer les $s_{a}$ dans les $C_{j}$ et l'on a plusieurs méthodes pour obtenir les valeurs numériques $x_{a}$ pour les stimuli et $x_{j}$ pour les bornes entre deux catégories. C'est le cas de la loi des jugements catégoriels de Thurstone et Saffir [Torgerson, 1958 ; Bonnet, 1986], puis du mesurage des intervalles successifs [Adams, Messick, 1958 ; Maurin, 2009].

- On peut mettre également dans ce panorama l'estimation de la grandeur, magnitude estimation, développée par Stevens [Stevens, 1969]. Cette fois-ci le sujet exposé à un stimulus $s_{a}$ doit fournir une réponse numérique $x_{a}$ évaluée par comparaison à la réponse $x_{\text {ref }}$ correspondant à un stimulus de référence $s_{\text {ref }}$ de telle sorte que le rapport $x_{a} / x_{\text {ref }}$ soit proportionnel à l'intensité ressentie des sensations associées. Ce procédé empirique au départ a été ensuite repris par le mesurage [Krantz, 1972].

On note que dans les deux premiers cas on obtient une réponse numérisée après un traitement ad hoc tandis que l'on a d'emblée une réponse numérique dans le dernier, et que ces trois méthodes ont été reprises et intégrées dans la démarche générale du mesurage. À ce titre les valeurs que l'on en déduit appartiennent à $\mathrm{R}$ et sont mesurées sur une échelle dite d'intervalle avec les préférences et les intervalles successifs, tandis qu'avec l'estimation de la grandeur elles appartiennent à $\mathrm{R}^{+}$et sont mesurées sur une échelle dite de rapport [Roberts, 1979].

\subsection{LE CAS DU RECUEIL AVEC UN CURSEUR}

L'estimation de la grandeur et l'emploi d'un curseur sur $[0,1]$ ont en commun le fait que la réponse produit d'emblée un nombre, par exemple «Dans la méthode de cotation graphique le sujet doit cocher un point d'une droite représentant le continuum de jugement ; la distance par rapport à une origine servira de mesure » [Bonnet, 1986]. En l'occurrence il s'agit d'un nombre positif dans le premier cas et compris entre 0 et 1 dans le second quand on rapporte la distance à la longueur du continuum. La présence d'une borne inférieure au champ des réponses est un autre point commun, après quoi la présence d'une borne supérieure dans le cas d'un curseur introduit une différence importante. C'est cet encadrement des réponses entre deux bornes qui constitue la spécificité de ce procédé, et cela implique naturellement des différences quant au traitement des données en aval du recueil.

En effet si l'on peut faire des multiplications qui respectent la nature des données sur les nombres en provenance de l'estimation de la grandeur, il n'en est pas de même quand on est en présence d'une borne supérieure. Il se produit des effets de bord et les opérations numériques en vigueur pour les échelles de rapport et/ou d'intervalle ne peuvent convenir pour les nombres recueillis sur [0,1]. Ces effets s'opposent de facto à «l'hypothèse implicite ou explicite, que les jugements sont distribués sur une échelle 
dont les intervalles sont apparemment égaux » [Bonnet, 1986]. La même hypothèse figure également dans [Torgerson, 1958], mais comme elle précède la théorie du mesurage nous sommes amenés ici à prendre nos distances avec elle (cf. Annexe 1).

\subsection{POUR UNE NOUVELLE APPLICATION DU MESURAGE}

Dans le cas d'un curseur sur $[0,1]$ il faut ainsi pouvoir intégrer la présence des deux bornes. La théorie du mesurage ayant précisément pour objet d'apporter une représentation numérique en accord avec le mode de recueil employé, la théorie se doit, pour tout mode qu'elle n'a pas encore abordé, de développer une application nouvelle qui rende compte de son originalité. Et cela peut concerner notamment les outils mathématiques utilisés ainsi que la manière dont ils sont mis en œuvre. C'est d'une telle application nouvelle dont il est question ci-après pour les réponses recueillies sur $[0,1]$, et ne seraitce que pour marquer la différence sur le plan sémantique avec les pratiques en usage, ce procédé de recueil est appelé «par curseur » en lieu et place de «cotation graphique » ou autre rating scale.

Dans cette application nous soulignons la présence des bornes qui font la spécificité du recueil, et ce sont elles qui guident en partie le choix des outils introduits au $\S 4$. Nous avons aussi retenu de les expliciter au niveau des consignes à donner au sujet, et de lui préciser qu'il doit en tenir compte pour positionner sa réponse. Nous retrouvons là une situation assez générale en théorie du mesurage avec des aspects qui sont propres au volet des sciences humaines et d'autres qui le sont au volet mathématique. Dans la suite l'article développe davantage le second volet et n'aborde le premier que plus légèrement dans le $\S 4.2$.

\section{UN GROUPE DE TRANSFORMATIONS QUI LAISSENT LE SEGMENT $[0,1]$ INVARIANT}

En préalable ce paragraphe rassemble quelques rappels sur les outils mathématiques qui sont utilisés par la suite.

\subsection{LES FONCTIONS HOMOGRAPHIQUES}

Ces fonctions ont pour expression $y=\frac{a x+b}{c x+d}$ et dépendent de 4 paramètres, elles sont définies sur $\mathrm{R}$ hormis éventuellement $-d / c$, leur dérivée est du signe de $a d-b c$, et ce sont des applications bijectives de $\mathrm{R}$ dans $\mathrm{R}$ pour $a d-b c \neq 0$. On introduit les coordonnées homogènes $(X ; T), T \neq 0$, des points en géométrie projective, telles que $(X ; T)$ et $(\lambda X ; \lambda T)$ correspondent au même point de la droite pour tout $\lambda \neq 0$, et au point à l'infini de la droite avec $T=0$ [Félix, 1962]. En mettant les coordonnées $(X ; T)$ sous la forme vectorielle $\left(\begin{array}{c}X \\ T\end{array}\right)$ la fonction homographique qui transforme $x$ en $y$ prend la forme matricielle $\left(\begin{array}{l}y \\ 1\end{array}\right)=\left(\begin{array}{ll}a & b \\ c & d\end{array}\right)\left(\begin{array}{l}x \\ 1\end{array}\right)$ avec la matrice $M_{a b c d}=\left(\begin{array}{ll}a & b \\ c & d\end{array}\right)$ de déterminant $a d-b c$. Par la même occasion le produit de composition des deux fonctions homographiques $z=\frac{e y+f}{g y+h}$ et $y=\frac{a x+b}{c x+d}$ est une nouvelle fonction homographique 
associée à la matrice produit $M_{e f g h} M_{a b c d}$. La matrice $M_{1001}$ correspond à la fonction identité $y=x$, et par conséquent les fonctions homographiques forment un groupe isomorphe au groupe linéaire GL2 des matrices réelles $2 \times 2$ régulières.

On rappelle que les fonctions homographiques laissent invariant le birapport $\left(x_{1}, x_{2} ; x_{3}, x_{4}\right)=\frac{x_{3}-x_{1}}{x_{3}-x_{2}} / \frac{x_{4}-x_{1}}{x_{4}-x_{2}}$ de tout quadruplet de valeurs $x_{1}, x_{2}, x_{3}$ et $x_{4}$ [Félix, 1962], et l'on note que la fonction est croissante lorsque le déterminant $a d-b c$ est positif.

On peut s'assurer de la réciproque ; si $y=h(x)$ est une application définie monotone bijective sur $\mathrm{R}$ qui laisse tout birapport invariant, c'est une fonction homographique. En effet avec le quadruplet $x_{1}, x_{2}, x$ et $x_{4}$ et son transformé $y_{1}, y_{2}, y$ et $y_{4} \quad$ l'égalité des birapports $\left(y_{1}, y_{2} ; y, y_{4}\right)=\left(x_{1}, x_{2} ; x, x_{4}\right)$ donne $\frac{y-y_{1}}{y-y_{2}}=\frac{x-x_{1}}{x-x_{2}} \cdot \frac{y_{4}-y_{1}}{y_{4}-y_{2}} / \frac{x_{4}-x_{1}}{x_{4}-x_{2}}$, et l'application $h$ est homographique.

\subsection{LES FONCTIONS HOMOGRAPHIQUES CROISSANTES LAISSANT $[0,1]$ INVARIANT}

3.2.1. Dans un premier temps on prend deux nombres réels $A<B$ et on considère la transformation $f_{k}$ qui à tout $x$ de $\mathrm{R}$ fait correspondre $y$ avec la définition $\frac{y-B}{y-A}=k \frac{x-B}{x-A}$. Il s'agit d'une transformation homographique avec laquelle pour tout $\mathrm{k}$ fini non nul $\mathrm{x}$ et y tendent simultanément vers $A$, et vers $B$. Le déterminant est égal à $k(B-A)^{2}$ et pour $k$ positif $x$ et $y$ sont simultanément intérieurs ou extérieurs à $[A, B]$. On a ainsi pour $\mathrm{k}$ positif une fonction homographique croissante qui laisse le segment $[A, B]$ invariant.

3.2.2. La composition de $f_{k}$ avec l'application $f_{k^{\prime}}$ définie par $\frac{z-B}{z-A}=k^{\prime} \frac{y-B}{y-A}$ conduit à $\frac{z-B}{z-A}=k k^{\prime} \frac{x-B}{x-A}$, une application de même type avec le paramètre $k k^{\prime}$ positif. La fonction $f_{1}$ est l'élément neutre, $f_{1 / k}$ est l'inverse de $f_{k}$ et ces applications forment avec le produit de composition un groupe de transformations homographiques croissantes laissant $[A, B]$ invariant, en l'occurrence un groupe isomorphe au groupe multiplicatif sur $\mathrm{R}^{+}$.

3.2.3. On particularise ensuite au segment $[0,1]$ en prenant $A=0, B=1$ et le groupe des transformations homographiques $f_{01, k}$ d'équations $\frac{y-1}{y}=k \frac{x-1}{x}, \mathrm{k}>0, \mathrm{k}>0$. C'est, avec l'exemple évident des fonctions puissances $x^{\alpha}, \alpha>0$, un autre groupe de transformations croissantes pour la composition qui laissent $[0,1]$ invariant. 


\subsection{EN VUE D'UN THÉORÈME DE CARACTÉRISATION}

Ces rappels sur les fonctions homographiques ont pour objet d'expliciter un groupe de transformations croissantes, à savoir les $f_{k}, k>0$, qui laissent $[A, B]$, ou $[0,1]$, invariant afin de promouvoir une nouvelle application de la théorie du mesurage [Roberts, 1979].

Dans cette théorie les $f_{k}$ constituent un groupe de transformations dites admissibles, et la présence d'un tel groupe permet de caractériser une échelle de mesurage au cours de l'étape de la Caractérisation (uniqueness). Compte tenu du fait que les transformations ci-dessus utilisent le birapport et ses propriétés, il est proposé d'appeler cette échelle une « échelle anharmonique $A-B »$ pour le groupe des $f_{k}, k>0$, et une «échelle anharmonique $0-1$ » pour le groupe des $f_{01, k}, k>0$, en référence à Chasles qui préférait appeler un birapport un rapport anharmonique.

\subsection{QUELQUES PROPRIÉTÉS DES BIRAPPORTS}

On a noté que dans les transformations ci-dessus le birapport de tout quadruplet $\left(x_{1}, x_{2}, x_{3}, x_{4}\right)$ appartenant à $[A, B]$ est invariant. On note également que les birapports correspondants aux deux quadruplets $\left(x_{1}, x_{2}, x_{3}, x_{4}\right)$ et $\left(x_{1}, x_{2}, x_{4}, x_{5}\right)$ sont multiplicatifs, et vérifient notamment

$$
\left(x_{1}, x_{2} ; x_{3}, x_{4}\right)\left(x_{1}, x_{2} ; x_{4}, x_{5}\right)=\left(x_{1}, x_{2} ; x_{3}, x_{5}\right)
$$

comme cela résulte de la définition.

\subsection{UNE PROPRIÉTÉ DES $f_{k}$}

Dans la différence $y-x=\frac{(k-1)\left(x^{2}-(A+B) x+A B\right)}{x(1-k)-(A-k B)}$ le polynôme au numérateur est négatif sur $[A, B]$ et il en résulte que $y-x$ est du signe de $x-\xi$ en posant $\xi=(k B-A) /(k-1)$.

$\xi$ est une fonction homographique en $k$ qui est supérieure à $B$ pour $k>1$, et qui est comprise entre $A$ et $B$ pour $0<k<1$ avec $\xi(0)=A$. Par conséquent lorsque $x$ est compris entre $A$ et $B$ les deux différences $x-\xi$ et $y-x$ sont négatives pour $k>1$, et donc $y<x$; à l'inverse $x-\xi$ et $y-x$ sont positives pour $0<k<1$, et donc $y>x$.

\section{UNE ÉCHELLE DE MESURAGE POUR LES NOMBRES RELEVÉS SUR [0,1]}

\subsection{LA DÉMARCHE SPÉCIFIQUE DU MESURAGE}

De manière générale les observations des phénomènes sont qualitatives et prénumériques, elles vérifient aussi un certain nombre de relations entre elles et elles constituent de ce fait un Système Relationnel Empirique (SRE). L'objet du mesurage consiste à en obtenir une image numérique à l'aide d'une représentation dans un Système Relationnel Numérique (SRN), homomorphe au SRE en respectant toutes les relations de celui-ci. Cela passe par un théorème de représentation qui démontre l'existence d'une représentation entre le SRE et le SRN, avec pour hypothèses les relations du SRE mises sous la forme de conditions de mesurage (parfois appelées axiomes). De cette manière on est assuré que les images numériques qui résultent de la représentation sont en 
accord avec les relations expérimentales observées [Suppes, Zinnes, 1963 ; Roberts, 1979]. On peut aussi poser des conditions de mesurage afin d'explorer de manière théorique des possibilités de représentation, et naturellement ces conditions doivent être vérifiables en pratique de la part des observations pour lesquelles on recherche une représentation.

Le passage du SRE au SRN donne ainsi lieu à une assignation numérique, numerical assignment, ou encore à un plongement, embedding, dans un espace numérique. Et lorsque les observations sont d'emblée numériques comme c'est le cas avec une «fabrique directe » de nombres telle qu'un curseur sur $[0,1]$ ou l'estimation de la grandeur, on est immédiatement confronté à l'examen de conditions qui sont vérifiables de la part de ces nombres et qui conduisent à un théorème de représentation (cf. $§ 4.3$ ).

\subsection{LES GRANDES LIGNES D'UN PROTOCOLE EXPÉRIMENTAL ET COMMENTAIRES}

Ces grandes lignes font davantage partie du volet des sciences humaines, un volet qui d'une manière générale est moins abordé dans cet article.

4.2.1. Pour plus de généralité, on peut conserver des valeurs de bornes notées $A$ et $B$, en sachant au besoin que $A=0$ et $B=1$. Ces deux bornes doivent être explicitement mentionnées dans les consignes comme des stimuli de référence, et avec un stimulus unique $s$ le sujet qui fournit une réponse $x$ doit pour sa part envisager les différences $x-A$ et $B-x$.

Naturellement la problématique n'a d'intérêt que s'il y a plusieurs stimuli, et les bornes doivent jouer le même rôle pour chacun d'eux. En présence d'un couple $s_{i}, s_{j}$ le sujet doit ainsi positionner les réponses $x_{i}$ et $x_{j}$ et "gérer» quatre différences numériques. Lorsque par exemple $s_{i}$ est jugé «plus fort» que $s_{j}$ il est simultanément plus proche de $B$ et plus éloigné de $A$ que $s_{j}$, et le rapport $(x-A) /(B-x)$ est une fonction croissante de l'impression ressentie. On note que le rapport des rapports $\frac{x_{i}-A}{B-x_{i}} / \frac{x_{j}-A}{B-x_{j}}$ n'est autre que le birapport $\left(A, B ; x_{i}, x_{j}\right)=\frac{x_{i}-A}{x_{i}-B} / \frac{x_{j}-A}{x_{j}-B}$ des quatre arguments numériques $A, B, x_{i}$ et $x_{j}$, à savoir un outil qui précisément explicite la présence des deux bornes et qui s'introduit ici par l'intermédiaire d'une procédure de comparaison.

4.2.2. Les bornes extrémales figurent comme des stimuli de référence concrets ou bien plus abstraits, voire par défaut, comme par exemple le zéro dans l'estimation de la grandeur. Elles peuvent être concrètes dans le cas de stimuli connus, discrets et ordonnés, ou dans le cas de structures comme un treillis avec minorant et majorant universel [Barbut, Monjardet, 1970], et notamment dans un produit d'ordres totaux d'indices respectifs $i_{1} \ldots i_{p} \ldots i_{k}$, avec pour chacun d'eux des stimuli unidimensionnels ordonnés respectivement de $s_{i p m i n}$ à $s_{\text {ipmax }}$. On peut aussi laisser au sujet le soin de concevoir ces stimuli extrémaux dont, encore une fois, l'utilisation des continuums bornés a besoin, autant sur le plan technique que conceptuel.

4.2.3. Le birapport apparaît ici à l'occasion d'une présentation par paire ou en couple, en l'occurrence un procédé classique afin d'exprimer des préférences que l'on rencontre également dans la magnitude estimation qui procède par comparaison entre tout 
stimulus étudié et le stimulus de référence. À ce propos on ne peut que reconnaître une certaine valeur prémonitoire en psychophysique et en psychométrie au Duc de SaintSimon qui quelque part dans ses Mémoires déclarait «comme tout ne se mesure que par comparaisons, ... ». Sans doute était-il bien placé à la Cour de Versailles pour observer et «mesurer » les faveurs de tel ou tel courtisan.

4.2.4. En présence de plusieurs stimuli le protocole procède avec des triplets $\left(s_{i}, s_{j}, s_{l}\right)$ et leur présentation par couple $\left(s_{i}, s_{j}\right),\left(s_{j}, s_{l}\right),\left(s_{i}, s_{l}\right)$, comme pour la mise en œuvre d'un système BTL.

\subsection{UNE CONDITION DE MESURAGE}

On note $\eta_{i, j}$ le birapport qui intervient avec la présentation du couple $\left(s_{i}, s_{j}\right)$ et les réponses observées $\left(x_{i}, x_{j}\right)$ sur $[A, B]$. Compte tenu des trois présentations en couple provenant du triplet $\left(s_{i}, s_{j}, s_{l}\right)$, on pose la condition de mesurage suivante

$$
\eta_{i, j} \eta_{j, l}=\eta_{i, l} \quad\left[C_{c}\right]
$$

entre les birapports calculés individuellement à partir de chaque couple $\left(s_{u}, s_{v}\right)$. Cette condition énonce que les birapports sont multiplicatifs, et il s'agit bien d'une condition vérifiable au vu des $\left(x_{u}, x_{v}\right)$ observés sur chacun des couples.

On note que cette condition de nature multiplicative est analogue à celle du modèle de BTL qui fonctionne également à partir de présentations par paire issues d'un triplet.

\subsection{UNE REPRÉSENTATION (REPRESENTATION)}

Il est utile en premier de définir le « repositionnement » d'une valeur $y$.

Soient $x$ et $y$, si l'on change $x$ en $x^{\prime}$ le repositionnement de $y$ consiste à le remplacer par $y^{\prime}$ de telle sorte que la valeur du birapport est conservée, $\left(A, B ; x^{\prime}, y^{\prime}\right)=$ $(A, B ; x, y)$. Techniquement cela résulte d'une transformation homographique entre $y$ et $y^{\prime}$ (paramétrée par $x$ et $x^{\prime}$ ).

THÉORÈME 1. Quand la condition $\left[C_{c}\right]$ est vérifiée pour un triplet, il y a bijection entre les $s_{i}, s_{j}, s_{1}$ et le jeu de valeurs recueillies $x_{i}, x_{j}, x_{l}$ et repositionnées sur les réponses $x_{i}, x_{j}$ de la première présentation.

Soient les birapports $\eta_{i, j}=\left(A, B ; x_{i}, x_{j}\right)$ avec les curseurs du premier couple, $\eta_{j, l}=\left(A, B ; x_{j b}\right.$, $\left.\xi_{l}\right)$ pour le second et $\eta_{i, l}=\left(A, B ; x_{i b}, \xi_{l b}\right)$ pour le dernier. À l'aide du repositionnement on introduit les nouvelles valeurs $\left(A, B ; x_{j}, \xi_{l}^{\prime}\right)=\left(A, B ; x_{j b}, \xi_{l}\right)=\eta_{j, l}$ et $\left(A, B ; x_{i}, \xi_{l b}^{\prime}\right)=\left(A, B ; x_{i b}, \xi_{l b}\right)=\eta_{i, l}$. La condition $\left[C_{c}\right]$ entraîne que $\left(A, B ; x_{i}, x_{j}\right)$ $\left(A, B ; x_{j}, \xi_{l}^{\prime}\right)=\left(A, B ; x_{i}, \xi_{l b}^{\prime}\right)$, il en résulte que $\xi_{l}^{\prime}=\xi_{l b}^{\prime}$ que l'on peut ici noter $x_{l}$.

Les valeurs ainsi repositionnées sur les $x_{i}$ et $x_{j}$ recueillies à la première présentation de $s_{i}$ et $s_{j}$ conduisent bien à une bijection entre les deux triplets $s_{i}, s_{j}$, $s_{l}$ et $x_{i}, x_{j}, x_{l}$.

COMMENTAIRE. Il importe de ne pas se méprendre sur les deux relations $[M]$ et $\left[C_{c}\right]$ qui sont formellement analogues mais qui ne possèdent nullement la même portée. $[M]$ est 
une propriété multiplicative des birapports avec des arguments numériques définis, de valeurs connues ou non, tandis que $\left[C_{c}\right]$ est une condition qui, lorsqu'elle est vérifiée, permet de déduire l'existence de ces arguments. La différence entre ces deux relations illustre tout à fait la théorie du mesurage, celle-ci se soucie d'abord de l'existence des mesures (cf. $\left[C_{c}\right]$ ), avant que l'on puisse les impliquer dans les calculs (cf. $\left.[M]\right)$.

\subsection{LA CARACTÉRISATION (UNIQUENESS)}

Il arrive qu'à l'issue d'un théorème de représentation, on ait démontré l'existence de plusieurs représentations, et que l'on doive gérer cet excès de solutions. Généralement les représentations se déduisent les unes des autres par des transformations dites admissibles. Cette situation donne lieu à un second théorème, un théorème de caractérisation (uniqueness), qui est chargé de caractériser le type d'échelle de mesure qui est commun à l'ensemble des représentations. Lorsque les transformations admissibles forment un groupe, l'échelle résultante est dite régulière. Parmi les plus courantes et connues il y a l'échelle d'intervalle avec le groupe des transformations affines positives $a x+b, a>0$, et l'échelle de rapport avec le groupe des homothéties positives $a x, a>0$ [Suppes, Zinnes, 1963 ; Roberts, 1979].

Dans le cas présent les trois valeurs numériques précédentes $x_{i}, x_{j}$ et $x_{k}$ qui sont attachées au triplet $\left(s_{i}, s_{j}, s_{k}\right)$ dépendent notamment des valeurs $x_{i}$ et $x_{j}$ recueillies à l'occasion du premier couple $\left(s_{i}, s_{j}\right)$. Il n'y a donc pas unicité de la représentation, mais l'on peut énoncer le :

THÉORÈME 2. L'échelle de mesure précédente pour des nombres construits avec un curseur sur le segment $[A, B]$ est une échelle anharmonique $A$ - B.

En effet si l'on applique la transformation homographique croissante $\frac{y-B}{y-A}=k \frac{x-B}{x-A}, k>0$, ou $\frac{y-A}{y-B}=t \frac{x-A}{x-B}$ avec $t=1 / k$ qui laisse $[A, B]$ invariant, tous les birapports $\eta_{i, j}$ respectivement définis à partir $d u$ couple de recueils $x_{i}$ et $x_{j}$ attachés à la paire $\left(s_{i}, s_{j}\right)$ demeurent invariants, et la condition de mesurage $\left[C_{c}\right]$ demeure vérifiée à l'évidence.

On a rappelé que les fonctions homographiques croissantes qui laissent [A,B] invariant forment un groupe, c'est le groupe des transformations admissibles qui caractérise l'échelle de mesure appelée anharmonique A-B au \$3.3.

En conclusion les propriétés du birapport et des fonctions homographiques permettent d'aboutir à une échelle de mesure anharmonique $A$ - $B$ dans le cas général, et anharmonique 0 - 1 sur $[0,1]$. Le procédé qui «fabrique » des réponses numériques avec un curseur sur un continuum borné se prête ainsi tout à fait à un mesurage des stimuli.

\subsection{L'EXTENSION À $n$ STIMULI $s_{i} i=1 \ldots n$}

L'extension se fait progressivement avec l'adjonction d'un nouveau stimulus $s_{p+1}$ à un ensemble de $p$ autres qui sont déjà mesurés, $p \geq 3$, ce qui fait intervenir des nouveaux triplets formés avec chacun des couples des stimuli qui précèdent.

THÉORÈME 3. Si un stimulus $s_{p+1}$ vérifie la condition $\left[C_{c}\right]$ avec un couple $\left(s_{i}, s_{j}\right)$ extrait d'un ensemble déjà mesuré, $s_{p+1}$ est mesuré sur la même échelle anharmonique que les stimuli de l'ensemble. 
Pour commencer on simplifie la notation $\left(A, B ; x_{i}, x_{j}\right)$ des birapports par $\left(x_{i}, x_{j}\right)$ car les bornes demeurent les mêmes. Dans ces conditions l'ensemble des valeurs $x_{i}$ correspondant au p-uplet des stimuli mesurés vérifient les relations $[M]\left(x_{i}, x_{j}\right)\left(x_{j}, x_{l}\right)=$ $\left(x_{i}, x_{l}\right), i, j, l=1 \ldots p(\mathrm{cf} . \S 3.4)$.

Si la condition $\left[C_{c}\right]$ est vérifiée par hypothèse pour le triplet $\left(s_{i}, s_{j}, s_{p+1}\right)$, il existe une représentation numérique $x_{p+1}$ de $s_{p+1}$ qui vérifie la relation $\left(x_{i}, x_{p+1}\right)\left(x_{p+1}, x_{j}\right)=$ $\left(x_{i}, x_{j}\right)$.

En multipliant les deux termes par $\left(x_{k}, x_{i}\right)$ à gauche, et par $\left(x_{j}, x_{l}\right)$ à droite on en déduit

$$
\left(x_{k}, x_{i}\right)\left(x_{i}, x_{p+1}\right)\left(x_{p+1}, x_{j}\right)\left(x_{j}, x_{l}\right)=\left(x_{k}, x_{i}\right)\left(x_{i}, x_{j}\right)\left(x_{j}, x_{l}\right),
$$

c'est-à-dire $\left(x_{k}, x_{p+1}\right)\left(x_{p+1}, x_{l}\right)=\left(x_{k}, x_{l}\right)$ d'après les relations $[M]$.

Par conséquent c'est avec la même valeur $x_{p+1}$ que $s_{p+1}$ vérifie $\left[C_{c}\right]$ avec tous les couples $\left(x_{k}, x_{l}\right)$ du p-uplet de stimuli $s_{i} i=1 \ldots p$.

\subsection{LE CAS PARTICULIER DE $[0,1]$}

Sur $[0,1]$ le birapport devient $\left(0,1 ; x_{i}, x_{j}\right)=\frac{x_{i}}{x_{i}-1} / \frac{x_{j}}{x_{j}-1}$ ou encore $\frac{x_{i}}{1-x_{i}} / \frac{x_{j}}{1-x_{j}}$. Par analogie avec les probabilités $p$ sur $[0,1]$ et leur cote $(o d d)$ associée $p /(1-p)$ on désigne ici également le rapport $x /(1-x)$ comme la cote de la valeur $x$. À l'aide de cette extension de terme assez naturelle, le birapport qui intervient dans le mesurage précédent est aussi le rapport de cotes (odds ratio) des nombres recueillis pendant l'expérience de la présentation de stimuli par couple.

\subsection{UN AMÉNAGEMENT NOUVEAU ET IMPORTANT}

Le recueil avec un curseur d'une réponse bénéficie d'une circonstance très favorable qui le distingue des autres procédés mentionnés, et notamment du BTL bien que celui-ci fonctionne également avec des comparaisons par paires extraites d'un triplet.

En effet on peut envisager de présenter simultanément le triplet $s_{i}, s_{j}$, $s_{l}$, et demander au sujet de les positionner en même temps sur $[A, B]$ ou sur $[0,1]$, en prenant au besoin le temps de la réflexion. Si tel est le cas la condition $\left[C_{c}\right]$ qui s'adresse aux trois birapports $\eta_{i, j}, \eta_{j, l}$ et $\eta_{i, l}$ est de facto vérifiée au titre des relations $[M]$ avec $x_{i}, x_{j}, x_{l}$, et la représentation en résulte aussitôt. On économise ainsi plusieurs étapes et l'on obtient d'emblée le mesurage des réponses $x_{i}$ sur une échelle anharmonique. L'aménagement est rendu possible puisque, sans compter le mesurage sur échelle anharmonique établi au préalable, on dispose ici d'un «mode de production » de plusieurs réponses numériques en même temps à partir d'une «fabrique » de nombres ; et cela ne peut être le cas pour les probabilités $p_{i j}$ du BTL.

Cela s'applique aussi à tout $\mathrm{n}$-uplet de stimuli multiples présentés simultanément. À partir d'un recueil des réponses $x_{i 1}, x_{i 2}, \ldots, x_{i n}$ en une seule fois les conditions $\left[C_{c}\right]$ sont de facto vérifiées en tant que relations $[M]$, et les $x_{i}$ sont sur la même échelle de mesure anharmonique. 


\subsection{COMPLÉMENT}

Cette démarche pour le mesurage de nombres recueillis sur $[0,1]$ paraît originale. Dans le même temps, en matière de recherche, on ne peut exclure d'envisager d'autres possibilités de représentation avec d'autres applications éventuellement en concurrence. Chacune d'elles possède ses propres conditions axiomatisées de mesurage, et si le cas échéant la question d'un choix se pose, il y a lieu de s'en remettre à la qualité du respect de ces conditions de la part des données pour valider la solution à retenir.

\section{QUELQUES OUTILS NUMÉRIQUES ET STATISTIQUES LIÉS À LA PERTINENCE (MEANINGFULNESS)}

D'une manière générale la pertinence s'applique aux énoncés numériques, numerical statements, qui sont établis à partir des valeurs mesurées, et pour cela elle fait intervenir le groupe des transformations admissibles qui sert à caractériser l'échelle de mesure [Roberts, 1979]. Dans le cas présent il s'agit du groupe anharmonique 0-1, et l'on suppose que toutes les mesures $x_{i}$ sont repositionnées, par exemple sur les valeurs du premier couple de stimuli présentés au sujet. À titre d'énoncés particuliers nous examinons ci-dessous quelques statistiques construites avec des $x_{i}$.

\subsection{LES BIRAPPORTS}

Le birapport $\eta_{i j}=\left(0,1 ; x_{i}, x_{j}\right)$ est invariant par l'action de toute transformation du groupe. Il s'agit ainsi d'un outil numérique invariant, et il en est de même naturellement pour toute fonction avec ces birapports comme arguments.

\subsection{LA MOYENNE HARMONIQUE}

Avec la transformation $y /(1-y)=t x /(1-x), t>0, \mathrm{du}$ groupe on a aussi $1 / y=(t-1) / t+1 / t x$. Il en résulte que si $m_{h x}$ est la moyenne harmonique de données $x_{i}$ définie par $1 / m_{h x}=1 / n \Sigma 1 / x_{i}$, la moyenne harmonique des transformées $y_{i}$ vérifie la relation $1 / m_{h y}=(t-1) / t+1 / t m_{h x}$.

Par conséquent la moyenne harmonique des $x_{i}$ se transforme comme le font les données $x_{i}$ pour toute transformation du groupe.

\subsection{DES RELATIONS ENTRE MOYENNES}

La cote $c=x /(1-x)$ vérifie $1 / c=1 / x-1$. On en déduit $1 / m_{h x}-1=1 / n \Sigma 1 / c_{i}$, par conséquent l'inverse de la cote de la moyenne harmonique des $x_{i}$ est la moyenne arithmétique de l'inverse des cotes des $x_{i}$.

\subsection{UNE CURIOSITÉ, UNE AUTRE MOYENNE}

i) Avec la transformation homographique précédente on a aussi $1 /(y-1)=(t-1)+t /(x-1)$. Il en résulte que si l'on pose la moyenne des $1 /\left(x_{i}-1\right)$, appelée par exemple la moyenne harmonique décalée de -1 , cette autre moyenne des $x_{i}$ se transforme également comme le font les $x_{i}$ avec le groupe de transformations admissibles. On peut la noter $\mu_{h x-1}$. 
ii) Avec la cote de $x$ on a $c-1=1 /(x-1)$; il en résulte encore que $1 / n \Sigma 1 /\left(x_{i}-1\right)=1 /\left(\mu_{h x-1}-1\right)$ est égale à la moyenne arithmétique des cotes $c_{i}$ décalée de -1 .

\section{AUTRES MESURAGES ASSOCIÉS}

Les nombres recueillis sur $[0,1]$, dûment mesurés sur une échelle anharmonique, ne sont pas très commodes à travailler, les moyennes présentées du $\S 5$ en témoignent. Cidessous nous entamons une seconde partie de l'article en explicitant de nouvelles échelles de mesure plus abordables avec les calculs ordinaires. Ces développements constituent une exploitation du mesurage des données recueillies avec un curseur ; ils présentent une portée pratique et statistique, $\S 7$, beaucoup plus importante que celle des nombres sur échelle anharmonique et cela ajoute un intérêt non négligeable à ce mode de recueil.

\subsection{LES COTES ET LEUR ÉCHELLE DE MESURE SUR R ${ }^{+}$}

Avec la terminologie du $\S 4.7$, un birapport est aussi un rapport de cotes (odds ratio) dans $\mathrm{R}^{+}$, par conséquent le mesurage du $\S 4$ conduit à un mesurage pour les cotes $c=x /(1-x)$ avec une échelle de rapport.

i) En effet la cote $c$ est une transformation bijective croissante de $[0,1]$ dans $\mathrm{R}^{+}$, et la condition $\left[C_{c}\right]$ qui concerne la multiplicativité des birapports exprime également la multiplicativité des rapports de cotes.

ii) Par ailleurs la transformation $y /(1-y)=t x /(1-x), t>0$, a pour effet de multiplier les cotes par un coefficient, il en résulte que les cotes sont mesurées sur une échelle de rapport.

On pourrait ainsi établir ici un second théorème de représentation et un second théorème de caractérisation pour le mesurage des cotes $c_{i}$ à partir de la même condition de mesurage $\left[C_{c}\right]$.

\subsection{LES LOGARITHMES DE COTE ET LEUR ÉCHELLE DE MESURE SUR R}

i) Le logarithme d'un birapport est le logarithme d'un rapport de cotes (log odds ratio) et c'est également la différence de deux logarithmes de cote, $\ln \left(0,1 ; x_{i}, x_{j}\right)=\ln c_{i}-\ln c_{j}$.

ii) Le logarithme de cote $\ln x /(1-x)$ est une transformation bijective croissante de $[0,1]$ dans $\mathrm{R}$, et la condition $\left[C_{c}\right]$ exprime aussi l'additivité des différences de logarithmes de cote. Dans ce cas la transformation $y /(1-y)=t x /(1-x), t>0$ a pour effet de translater tout logarithme de cote de la quantité $\ln t$.

iii) En mesurage les échelles pour lesquelles les translations $x+C$ constituent les transformations admissibles sont les échelles de différence définies sur R [Roberts, 1979]. À ce titre il est important de remarquer que le logarithme transforme les valeurs sur une échelle de rapport avec les homothéties positives admissibles $a x, a>0$, en valeurs sur une échelle de différence avec les translations $\ln x+\ln a$.

iv) Par conséquent les logarithmes de cote $\ln c=\ln x /(1-x)$ sur $\mathrm{R}$ sont mesurés sur une échelle de différence ; et par la même occasion les différences de logarithmes de 
cote $\ln c_{i}-\ln c_{j}$ sont mesurées sur l'échelle absolue (l'échelle absolue est l'échelle pour laquelle le groupe des transformations admissibles est réduit à l'application identité). Cela résulte aussi du caractère invariant du logarithme du birapport associé (cf $\S 5.1$ ).

v) De la même façon que pour la cote on pourrait ici établir de nouveaux théorèmes de représentation et de caractérisation pour le mesurage du logarithme de cote en prenant l'additivité des différences de logarithmes de cote comme nouvelle condition de mesurage.

vi) Au niveau des statistiques usuelles, la moyenne arithmétique de logarithmes de cote est une statistique sur la même échelle de différence que les logarithmes de cote, et l'on remarque qu'elle est aussi le logarithme de la moyenne géométrique des cotes $\ln \left(\Pi c_{i}\right)^{1 / n}$.

Il résulte de tout cela qu'à côté de l'échelle anharmonique pour les réponses $x$ du curseur qui est un peu ésotérique, on dispose de deux autres échelles de mesure, une échelle de rapport et une échelle de différence auxquelles on est mieux accoutumé.

\section{DES STATISTIQUES AVEC CES NOUVELLES ÉCHELLES}

Les traitements statistiques font partie des énoncés numériques que l'on établit avec les valeurs mesurées, et ils sont pertinents lorsqu'ils mettent en œuvre des formulations invariantes par le groupe des transformations admissibles. C'est notamment ce qui se passe avec les échelles de rapport et de différence qui offrent de nombreuses opportunités. Ci-dessous nous en mentionnons quelques-unes.

\subsection{DES TRAITEMENTS STATISTIQUES SUR LES COTES}

\subsubsection{Différences et variances}

Puisque les cotes $c=x /(1-x)$ sont mesurées sur une échelle de rapport, la moyenne arithmétique d'une population de $c_{i}$ est sur la même échelle de rapport que les $c_{i}$, au même titre que toute différence $\Delta c_{i l}=c_{i}-c_{l}$. Il en résulte que la variance de toute population de $c_{i}$ est également sur une échelle de rapport; et ceci est encore vérifié pour toute sous-population de cotes.

Par conséquent si l'on a des stimuli $s_{i}$ qui sont répartis entre plusieurs modalités, les modalités d'un facteur expérimental par exemple, on peut classiquement faire la décomposition de la variance totale des cotes $c_{i}$ avec un terme de variance intermodalités et un terme de variance intra-modalités. En outre le rapport de ces deux parts de variance est un nombre sur l'échelle absolue.

\subsubsection{Les facteurs d'un plan d'expérience}

On peut de même envisager des stimuli $S_{i 1, i 2, \ldots, i k}$ qui dépendent de plusieurs facteurs, ainsi qu'un plan d'expérience (complet, incomplet, équilibré, avec ou sans répétitions, ...) avec les modalités de ces différents facteurs comme on le fait de manière ordinaire dans tout contexte statistique de plan d'expérience [Dugué, Girault, 1969]. Dans ces conditions l'indice des stimuli est multiple $\{i\}=\left(i_{1}, i_{2}, \ldots, i_{k}\right)$ et l'on 
peut sur les $c_{\{i\}}$ procéder aux décompositions quadratiques de la variance totale comme on le fait ordinairement quand on est en présence de plusieurs facteurs. Ces différentes parts de la variance des cotes sont ici sur une échelle de rapport, et leurs rapports entre elles sur l'échelle absolue. Par exemple, si l'on est dans le cas d'une structure de treillis produit d'ordres totaux, $\S 4.2 .2$, chacun de ces ordres peut précisément être un facteur.

\subsubsection{La présence de plusieurs sujets}

On a jusqu'à présent implicitement considéré que les stimuli $s_{\{i\}}$ et les mesures correspondantes $x_{\{i\}}$ et puis $c_{\{i\}}$ ne concernent qu'un seul sujet.

Dans le cas contraire, on obtient les mesures de cotes $c_{\{i\} j}$ avec l'indice $j$ qui s'applique au sujet. Il est assez naturel de procéder à des répétitions d'observations sur des sujets en psychophysique [Torgerson, 1958 ; Bonnet, 1986 ; Martin, 1997], mais dans le cadre du mesurage Roberts fait remarquer que chacun des sujets (ou membres de jury) mesure selon sa propre échelle et que cela complique fortement les énoncés pertinents que l'on peut établir [Roberts, 1979]. Précisément si la réponse est une mesure $u_{i}$ sur échelle de rapport, les $u_{i j}$ par sujet le sont aussi avec des transformations admissibles de la forme $a_{j} u_{i j}$; et dans ces conditions la moyenne arithmétique intersujets n'est pas pertinente (elle n'est pas invariante par l'action conjointe de ces différents groupes de transformation). Pour remédier à cet état de fait Roberts remarque que la moyenne géométrique des réponses de chaque sujet $\left(\Pi_{j} a_{j}\right)^{1 / n}\left(\Pi_{j} u_{i j}\right)^{1 / n}$ est sur une nouvelle échelle de rapport avec le coefficient multiplicatif commun $\left(\Pi_{j} a_{j}\right)^{1 / n}$.

Dans ces conditions on peut appliquer sur ces moyennes géométriques les décompositions de variance précédentes des $\S 7.1 .1$ ou $\S 7.1 .2$ quand il y a plusieurs facteurs $\left(i_{1}, i_{2}, \ldots, i_{k}\right)$.

\subsubsection{Un facteur sujet}

Cependant on peut aller au delà et résoudre d'une autre manière la difficulté qui est soulevée par la pluralité des échelles de rapport individuelles. Il suffit pour cela d'introduire explicitement un nouveau facteur, le facteur dont les modalités sont les différents sujets.

En effet on peut établir un calibrage des échelles de rapport de chaque sujet en posant un stimulus $s_{\{0\}}$ de référence. À partir des $\gamma_{\{i\} j}=a_{j} c_{\{i\} j}$ et en prenant la réponse à cette référence comme valeur commune $K$ on en déduit que les réponses pondérées $\gamma_{\{i\} j}=K c_{\{i\} j} / c_{\{0\} j}$ sont sur la même échelle de rapport pour tout sujet $j$. On peut en outre désigner éventuellement un sujet $j_{0}$ de référence et poser $K=c_{\{0\} j 0}$ pour les nouvelles mesures de cotes $\gamma_{\{i\} j}=c_{\{i\} j} c_{\{0\}_{j 0}} / c_{\{0\} j}$.

L'indice $j$ figure ainsi comme l'indice des modalités d'un nouveau facteur sur des mesures qui appartiennent à une échelle commune. Cela correspond formellement à un facteur supplémentaire que l'on peut appeler « facteur sujet » puisque ses modalités sont les différents sujets de l'enquête (ou du jury) pris individuellement. On peut aussi, éventuellement, répartir les sujets en diverses sous-populations comme l'âge, le sexe, etc., et en prenant les moyennes de réponses des sujets par sous-population introduire un facteur âge, un facteur sexe, etc. 
Dans tous les cas ci-dessus on se retrouve dans une situation statistique ordinaire pour l'analyse des résultats d'un plan d'expérience (avec ici un facteur supplémentaire après le calibrage des sujets). Avec les analyses statistiques de cette sorte on entre dans une autre problématique, et l'on sait bien par exemple que l'une des limites pratiques à ces traitements est le nombre de facteurs, de modalités et de répétitions, etc. [Dugué, Girault, 1969 ; Chapouille, 1973].

\subsection{DES TRAITEMENTS STATISTIQUES SUR LES LOGARITHMES DE COTES}

On a d'autres opportunités statistiques en aval du mesurage avec les logarithmes de cote $\ln c=\ln x /(1-x)$ notés $z$ pour simplifier. On reprend les mêmes rubriques qu'au $§ 7.1$.

\subsubsection{Différences et variances}

La moyenne arithmétique d'une population ou sous-population de $z_{i}$ est sur la même échelle de différence, et toute différence $\Delta z_{i l}=z_{i}-z_{l}$ est un nombre absolu. Il en est ainsi de même de la variance de toute population ou sous-population de $z_{i}$.

Par conséquent avec des stimuli répartis entre plusieurs modalités, on obtient également la décomposition de la variance totale avec les deux termes de variance intermodalités et intra-modalités; le rapport des deux est a fortiori un nombre absolu.

\subsubsection{Les facteurs d'un plan d'expérience}

On retrouve avec les $z_{\{i\}}$ la même possibilité multifactorielle qu'en $\S 7.1 .2$, les différentes parts d'une décomposition quadratique de variance sont ici des nombres absolus ainsi que leurs rapports.

\subsubsection{La présence de plusieurs sujets}

Les $z_{\{i\} j}=\ln c_{\{i\} j}$ sont mesurés sur des échelles de différence différentes d'un sujet à l'autre, mais en aménageant la solution de Roberts rappelée au $§ 7.1 .3$, leur moyenne arithmétique $1 / n \Sigma_{j} z_{\{i\}_{j}}$ est sur une nouvelle échelle de différence. On peut ensuite leur appliquer les mêmes décompositions de variance que ci-dessus au $\S$ 7.1.1 et $\S$ 7.1.2.

\subsubsection{Un facteur sujet ou membre de jury}

On résout également la difficulté de la pluralité des échelles de différence des sujets en adaptant le calibrage des réponses du $§ 7.1 .4$, avec un stimulus de référence $s_{\{0\}}$ et un sujet de référence $j_{0}$. On a ainsi des valeurs translatées $\zeta_{\{i\} j}=\ln \gamma_{\{i\} j}=\ln a_{j}+z_{\{i\} j}$ de telle sorte que $\zeta_{\{0\} j}=\ln K$ pour tous les sujets. Il en résulte que les $\zeta_{\{i\} j}=\ln K+z_{\{i\} j}-z_{\{0\} j}$ sont sur la même échelle de différence, et l'on peut au besoin introduire un sujet de référence $j_{0}$ pour avoir $\xi_{\{i\} j}=z_{\{i\} j}+\left(z_{\{0\} j 0}-z_{\{0\} j}\right)$ avant de procéder à des décompositions de variance. Cela se traduit par l'introduction du facteur sujet supplémentaire, ou d'un facteur par les modalités de sous-population, âge, sexe, etc.

Comme indiqué dans l'introduction, $\S 1$, l'article est le fruit de quelques discussions à propos de la pratique du curseur sur un continuum borné, et il n'y a pas pour l'instant de jeu de données pour mesurer les réponses sur échelle anharmonique ni pour les exploiter de cette manière. Cela demeure pour l'instant la présentation d'un 
modèle formel, avec diverses possibilités de poursuite statistique étendues et pertinentes.

\section{COMMENTAIRES SUR LE LOGARITHME D'UN BIRAPPORT}

La présence d'un birapport, de son logarithme et du logarithme des cotes est l'occasion de quelques commentaires «culturels » (sans exhaustivité ni prétention) pour montrer que ces outils se rencontrent en d'autres circonstances.

\subsection{LA GÉOMETRIE DE POINCARÉ}

C'est une géométrie non euclidienne dans le demi-plan complexe de partie imaginaire positive [Valiron, 1955]. Dans ce demi-plan la distance entre deux points $M_{1}$ et $M_{2}$ s'exprime par le logarithme d'un birapport dans lequel figurent $M_{1}, M_{2}$ ainsi que deux autres points en limite du demi-plan de manière analogue aux bornes $A$ et $B$ ci-dessus. Cela donne une idée du potentiel de cet outil.

\subsection{LA FONCTION DE LIEN (LINK FUNCTION) DU MODÈLE LOGISTIQUE}

Le Modèle Linéaire Généralisé (GLM), consiste à transformer des probabilités sur [0,1] en variables transformées sur $\mathrm{R}$ afin de les faire rentrer dans le schéma explicatif ordinaire d'une combinaison linéaire de variables indépendantes $\Sigma a_{i} X_{i}$ [Mc Cullagh, Nelder, 1989]. En la matière on prend pour fonction de lien la réciproque $F^{-1}$ de la fonction de répartition $F$ d'une loi de variable aléatoire définie sur R. Beaucoup de $F$ peuvent être envisagées et en pratique la fonction de répartition de la loi normale est exclue sans doute pour la raison qu'elle ne dispose pas d'une formulation analytique explicite.

La plus utilisée est sans conteste celle de la loi logistique avec $p=F(x)=1 / 2(1+$ th $x)$ et donc $x=F^{-1}(p)=1 / 2 \ln p /(1-p)$. C'est une expression particulièrement simple qui doit pour l'essentiel expliquer le succès rencontré par le modèle logistique en GLM, et l'on y rencontre la cote d'une probabilité ainsi que son logarithme.

\subsection{LA VITESSE RELATIVISTE D’EINSTEIN}

Dans la cinématique galiléenne les vitesses $v$ sont additives sur $\mathrm{R}$, mais dans la relativité restreinte la vitesse qui est comprise dans l'intervalle $[-c, c]$ avec $c$ la vitesse de la lumière ne peut plus l'être. Avec sa nouvelle opération «somme relativiste » de vitesses $\frac{v_{1}+v_{2}}{1-v_{1} v_{2} / c^{2}}$ Einstein montre que les transformées Argth $v / c=1 / 2 \ln (c+v) /(c-v)$ sont additives sur $\mathrm{R}$; c'est en substance le $F^{-1}$ de la loi logistique adaptée au segment symétrique $[-c, c]$ et son équivalent sur le logarithme de la cote après la réduction $\operatorname{par} c$.

Tout cela peut donner lieu à une suite qui a des retombées en mesurage (cf. Annexe 2). 


\section{CONCLUSION}

L'objectif de cet article est de formaliser les recueils de réponses faits avec la position d'un curseur sur un continuum borné $[A, B]$ ou $[0,1]$ de manière conforme à l'esprit du mesurage. À l'occasion de cette application nouvelle de la théorie il y a naturellement lieu de tenir compte des deux bornes et de leur effet de bord, et le birapport qui s'introduit à l'aide d'un couple de stimuli se prête bien au développement. D'une part, sur le plan de la mise en ouvre cela implique des consignes à donner au sujet quant à la manière d'appréhender les bornes au cours de sa réponse. De l'autre, sur le plan mathématique les propriétés du birapport et des fonctions homographiques conduisent à des démonstrations de représentation et de caractérisation relativement simples.

Le résultat peut au premier abord paraître quelque peu trivial car il ne fait qu'exploiter des propriétés d'outils mathématiques bien connus par ailleurs. En outre le fait de recycler dans son propre cadre de travail des résultats mathématiques établis en d'autres circonstances, éventuellement délaissé(s) et méconnu(s) ... dans quelque arrière salle du magasin mathématique [Le Lionnais, 1962], peut masquer en partie la présence de la théorie du mesurage. C'est aussi le cas par exemple des équations fonctionnelles [Aczél, 1966], de la cancellation de Thomsen [Luce, Tuckey, 1964 ; Roberts, 1979] ou d'un théorème de Mardia et de Plackett [Maurin, 1989]. Mais c'est également un grand mérite que de reprendre des résultats existants afin de les mettre au service de la représentation numérique pour des disciplines dans lesquelles il faut maîtriser un certain tropisme vers le nombre et la tentation de s'en servir sans trop de précautions. À ce titre on ne doit pas se méprendre à propos de l'apparente banalité éventuelle des outils mis en œuvre, et l'on aboutit ici à un nouveau type d'échelle de mesurage, une échelle dite anharmonique.

Après quoi ce mesurage présente un autre avantage, celui de conduire à des échelles de mesure associées qui offrent de nombreuses possibilités de traitements statistiques usuels. C'est un complément important qui accompagne le mesurage des réponses données sur un continuum borné, et cela renforce l'intérêt du birapport et des fonctions homographiques exhumés pour la circonstance.

Post-scriptum. Ce résultat n'aurait pas eu lieu sans le hasard d'une rencontre avec Arnaud Trollé ni les riches et nombreuses discussions qui ont suivi.

Remerciements aux relecteurs qui ont lu le projet d'article avec beaucoup d'attention, leurs remarques ont permis d'apporter des précisions et compléments qui contribuent à une présentation plus claire et structurée de ce nouveau type d'échelle.

\section{BIBLIOGRAPHIE}

ACZÉL J. (1966), Lectures on functional equations and their applications, vol. 19, New York, London, Academic Press.

ADAMS E., MESSICK S. (1958), “An axiomatic formulation and generalisation of successive intervals scaling”, Psychometrika 23(4), p. 355-368

BARBUT M., MONJARDET B. (1970), Ordre et classification, tome 1, Paris, Hachette Université.

BONNET C. (1986), Manuel pratique de psychophysique, Paris, A. Colin.

CHAPOUILle P. (1973), Planification et analyse des expériences, Paris, Masson \& Cie.

DEBATY P. (1967), La mesure des attitudes, Paris, Presses Universitaires de France. 
DUGUE D., GIRAUlT M. (1969), Analyse de variance et plan d'expérience, Paris, Dunod.

FELIX L. (1962), Exposé moderne des mathématiques élémentaires, Paris, Dunod.

KRANTZ D. H. (1972), “A theory of magnitude estimation and cross modality matching”, Journal of Math. Psychol. 9, p. 168-199

LE LIONNAIS F. (1962), «Les mathématiques et les sciences de la nature », F. Le Lionnais (éd.), Les grands courants de la pensée mathématique, Paris, A. Blanchard, p. 389-392.

LUCE R.D., TUCKEY J.W. (1964), "Simultaneous conjoint measurement, a new type of fundamental measurement", Journal of Math. Psych 1, p. 1-27

MC CULLAGH P., NELDER J.A. (1989), Generalised linear models, 2e ed., Chapman and Hall.

MARTIN O. (1997), La mesure de l'esprit, Paris, l'Harmattan.

MAURIN M. (1989), «Le mesurage des intervalles successifs doubles, une application des distributions bivariées à marges données », Mathématiques Informatique et Sciences humaines 108, p. 23-34.

MAURIN M. (2009), « Lorsque l'utilité, la gêne ou le confort sont recueillis sur une échelle de catégories : l'interaction dans le contexte multivarié », Mathématiques et Sciences humaines 188, p. 5-39.

MICHELL J. (1990), An introduction to the logic of Psychological Measurement, Hillsdale (NJ), Lauwrence Erlbaum Associates Publishers.

PARAIN-VIAL J. (1981), «Introduction », J. Parain-Vial (éd), Les difficultés de la quantification et de la mesure, Paris, Maloine S-A, p. 5-11.

POIRIER R. (1981), « Mesure et réalité de l'objet », J. Parain-Vial (éd.), Les difficultés de la quantification et de la mesure, Paris, Maloine S-A, p. 275-293.

ROBERTS F.J. (1979), Measurement theory, Reading (MA), Addison Wesley P. Co.

STEVENS S. S. (1969), «Le quantitatif et la perception », Bulletin de Psychologie 276 tome XXIII, p. 696715.

SUPPES P., ZINNES J.L. (1963), "Basic measurement theory”, R.D. Lace, R.R. Bush, E. Galanter (eds.), Handbook of Math. Psychology 56, J. Wiley and sons, p. 1-76.

TORGERSON W. S. (1958), Theory and methods of scaling, J. Wiley and sons.

VALIRON G. (1955), Théorie des fonctions, Paris, Masson. 


\section{ANNEXE 1}

\section{À PROPOS DE «L'ÉGALITÉ DES INTERVALLES »}

Ce n'est pas la première fois que l'hypothèse des intervalles égaux ou apparemment égaux est malmenée ou reconduite au placard.

On rencontre en effet, sans aucune exhaustivité historique, une situation analogue au sujet des intervalles apparemment égaux [Debaty, 1967].

En effet au cours de sa carrière Thurstone s'est intéressé à la mesure des attitudes et à leur classement par des juges dans des intervalles apparemment égaux. Mais étant prolixe, il a développé en parallèle la loi des jugements comparatifs, puis en a déduit la loi des jugements catégoriels à partir de réponses données sur une échelle de catégories ordonnées au début des années 30 [Torgerson, 1958 ; Bonnet, 1986]. Par la suite Adams et Messick ont intégré la loi des jugements catégoriels dans le formalisme de la théorie du mesurage en développant le mesurage des intervalles successifs [Adams, Messick, 1958 ; Suppes, Zinnes, 1963 ; Maurin, 2009].

D'une part les intervalles apparemment égaux se sont révélés assez laborieux à mettre en oeuvre, avec des end effects [Debaty, 1967], mais pour l'essentiel cette approche est rendue obsolète par le mesurage des intervalles successifs. En effet lorsque les données provenant d'une échelle catégorielle vérifient les conditions d'Adams et Messick, les différentes attitudes-stimuli sont mesurées sur une échelle d'intervalle, de même que les bornes entre les catégories (sur la même échelle). À ce titre on en déduit immédiatement les différences de valeur après mesurage entre les attitudes, et cette autre méthode résout ainsi plus directement la question qui a conduit à poser les intervalles apparemment égaux. 


\section{ANNEXE 2 \\ UN APARTÉ SUR L'ADDITIVITÉ, LA SIGNIFIANCE, LA PERTINENCE}

On a vu dans le texte trois exemples dans lesquels figurent le logarithme des cotes ou encore des argth, et cela permet de développer quelques considérations sur l'additivité des nombres et le bien fondé de son emploi.

1 - EN PHYSIQUE ET MÉCANIQUE (cf. § 8.3), le principe de relativité galiléenne énonce qu'avec des repères bien choisis (galiléens) la vitesse est additive et que la somme $v_{1}+v_{2}$ est elle-même interprétée comme une vitesse, celle d'un mobile existant mais rapportée à un autre repère que le repère initial. Ce n'est plus possible en relativité restreinte car les vitesses appartiennent au fermé $[-c, c]$, mais les développements

d'Einstein dans le cadre de nouveaux principes démontrent la nouvelle composition $\frac{v_{1}+v_{2}}{1-v_{1} v_{2} / c^{2}}$, c'est-àdire l'additivité des transformées argth v/c des vitesses galiléennes.

Dans les deux cas cette description du monde physique est due à des énoncés provenant d'observations scientifiques dûment constatées en leur temps, et elle souscrit à une exigence de ressemblance,

à la différence des mathématiques qui pratiquent parfois l'art pour l'art et le non-figuratif, la physique est essentiellement figurative, elle cherche la ressemblance, ... [Poirier, 1981].

La mesure, depuis Eudoxe, consiste à attribuer des nombres aux choses suivant certaines règles. Tout procédé de mesure est donc une mise en correspondance de propriétés, de relations entre propriétés expérimentalement établies et les propriétés des nombres [Debaty, 1967].

Les principes sont à la physique ce que sont les axiomes aux mathématiques, et la recherche de cette représentativité cinématique se fait dans le cadre de principes qui évoluent lorsque la vision de la physique s'affine, l'additivité des argth en résulte.

Cette qualité voulue de la ressemblance avec les propriétés expérimentales observées pourrait être appelée signifiance ; les opérations numériques (et analytiques) y acquièrent un sens vis-à-vis des phénomènes représentés.

2 - DANS LE CADRE DU MODÈLE LINÉAIRE GÉNÉRALISÉ (cf. § 8.2), on introduit des transformées $F^{-1}(p)$ qui ont pour objet de « décloisonner » un argument naturellement compris entre 0 et 1 . Par la suite, dans le cadre de l'art pour l'art qui respecte en priorité la cohérence des opérations mathématiques il est tout à fait possible d'appliquer la méthode et le formalisme de la régression linéaire en utilisant les opérations sur les nombres réels.

L'additivité des argth figure dans le modèle particulier de la loi logistique, et elle intervient au titre des calculs ordinaires sur les arguments numériques du GLM. Il n'est cependant pas assuré que les soucis de la représentativité des opérations et de l'interprétation des résultats soient les préoccupations phares du GLM, une remarque qui pourrait s'appliquer aussi au cas du modèle linéaire classique avec une variable à expliquer $Y$ qui est d'emblée sur R.

Un aparté dans l'aparté. Naturellement ces calculs et leurs règles sont définis bien en amont dans le cadre des mathématiques, et avec la familiarité et la force de l'habituation ils peuvent être considérés comme allant de soi. Par exemple Michell note que

Many psychologists, confident that their freedom to compute was as inalienable as their freedom to speak, took up arms in the cause of computational libertarianism [Michell, 1990]

et il évoque à l'occasion Lord et Burke, tandis que Martin reprend une déclaration de Lord « les nombres ne savent pas d'où ils viennent » afin d'illustrer ce courant permissif et libéral [Martin, 1997]. 
3 - LE MESURAGE DANS R du $§ 6.2$ avec une échelle de différence pour les logarithmes de cote de $x$ relève d'une troisième situation. Pour simplifier on pose $z=\ln x /(1-x)=2 \operatorname{Argth}(2 x-1)$; la qualité d'échelle de différence provient d'un théorème de caractérisation à l'aval d'un théorème de représentation selon les prescriptions de la théorie du mesurage. Dans ce cadre les $z_{i}$, images numériques des stimuli $s_{i}$, sont soustractives dans le sens que toute différence $\Delta z_{i j}=z_{i}-z_{j}$ est invariante par l'action du groupe des transformations admissibles de $\mathrm{R}$ dans $\mathrm{R}$ qui caractérise l'échelle obtenue. Certes la somme des $z_{i}$ est possible comme pour les $F^{-1}(p)$ du GLM, mais on peut en outre s'assurer ici que la moyenne arithmétique $m_{z}=1 / n \Sigma z_{i}$ est sur la même échelle de différence que les $z_{i}$, et en déduire notamment que des différences $m_{z}-z_{i}$ sont également invariantes par l'action de ce groupe.

La notion de pertinence vient après celle de la caractérisation, elle s'applique aux énoncés dont la valeur logique de ce qu'ils expriment est invariante par l'action du groupe des transformations admissibles. À ce titre la moyenne arithmétique des $z_{i}$ intervient dans des énoncés pertinents par l'intermédiaire de différences.

La signifiance et la pertinence ont ainsi en commun la ressemblance entre ce que l'on observe des phénomènes et leurs modes de traduction numérique. La signifiance fait en sorte de respecter les principes, et la pertinence de respecter l'action du groupe des transformations admissibles qui rendent compte des propriétés qualitatives des observations. 